\title{
Le jeu de mots dans la dramaturgie d'avant- garde des années 1950 : les exemples de Ionesco et de Tardieu
}

Résumé : Le jeu de mots, vu dans la perspective large de la nature du jeu - la création d'une illusion amusante et reposante en-dehors de la vie réelle -, signifie un écart par rapport à la convention générale et à la visée utilitaire de la langue, un abri, fondé sur la plaisanterie et le divertissement, contre le sérieux et la banalité des usages. Employé dans le théâtre comique traditionnel (d'inspiration aristotélicienne), le jeu de mots y renforce les effets illusionnistes en soulignant le comique d'idée, de caractère ou de situation. Le théâtre d'avant-garde des années 1950, libéré du souci de la mimésis, délivre le jeu de mots de son attachement servile à l'illusion vraisemblable et à la connivence dramatique que l'auteur cherchait auprès de son public, lui permet ainsi d'accéder à une forme de gratuité et une liberté totale - ce qui le transforme en même temps en un catalyseur de l'autonomisation du théâtre. Eugène Ionesco et Jean Tardieu sont tous deux des jongleurs de mots dans la lignée de Jarry; ils partagent le rêve d'avant-garde d'un théâtre abstrait, tout en adoptant, à l'égard du langage et de l'esthétique dramatique, deux attitudes d'après-guerre fort différentes - celle de rupture et celle de réconciliation. Ionesco œuvrait au dysfonctionnement du langage et à l'échec de la communication, tandis que Tardieu exploitait la fantaisie burlesque et la potentialité musicale de la langue. Les jeux de mots du premier, faisant fi de la logique et de la raison, relèvent de l'esprit anarchique qui fonde la plaisanterie et revêtent une fonction critique; ceux du second, explorant la dimension ludique et poétique des mots, s'approchent du principe esthétique du divertissement et revêtent une fonction poïétique. Une comparaison de ces deux types de jeux de mots, sur les plans paradigmatique et discursif, éclairera le rôle essentiel du jeu de mots dans le renouvellement du langage dramatique et de la vision générale de la langue au milieu du siècle dernier.

Mots clés : 1950, comédie du langage, connivence, divertissement, Eugène Ionesco, illusion, Jean Tardieu, jeu de mots, plaisanterie, théâtre d'avant-garde, tragédie du langage 


\section{Le jeu de mots et son rapport au théâtre}

\subsection{L'essence et les fondements du jeu de mots au sens large}

Si le jeu de mots, dans ce contexte théâtral, est d'emblée arraché à son sens strict ${ }^{1}$, c'est qu'il est souhaité d'aller au-delà de son utilité rhétorique dans un acte communicatif et du parti pris de son esprit non-sérieux, de son improductivité et de sa place mineure dans la littérature. Afin de restituer le jeu de mots dans sa complexité et sa plénitude, un passage par la notion de jeu s'avère nécessaire.

Huizinga, un des premiers à théoriser le jeu et sa fécondité dans le domaine culturel, situe cette « action libre, [...] dénuée de tout intérêt matériel et de toute utilité » (Huizinga 1951 : 31) à la base de l'ensemble de la culture (la liturgie, la compétition, la poésie, la philosophie, les arts voire les institutions juridiques) et souligne la distance que tout jeu crée, du fait de sa gratuité et de sa limite spatio-temporelle, avec la vie courante. Mettant l'accent lui aussi sur l'écart entre le jeu et le quotidien, Winnicott accorde au premier un rôle capital dans l'affirmation de soi et le développement de la créativité (Winnicott 1975 : 110). Quant à Roger Caillois, en affirmant à la fois que «tout jeu est système de règles » et qu'il entraîne "une atmosphère de délassement ou de divertissement» (Caillois $1967: 13$ et 9), il le situe entre deux pôles antagonistes et complémentaires - paidia et ludus, autrement dit « la puissance primaire d'improvisation et d'allégresse » et « le goût de la difficulté gratuite » (Caillois 1967 : 7576). Ceci revient à considérer la liberté et la contrainte comme deux attributs de la même notion, et à admettre que la règle, arbitrairement établie mais rigoureusement respectée, distingue à elle seule l'ordre du jeu de l'ordre de la vie et assure le fonctionnement de l'univers ludique. Huizinga et Caillois ont tous les deux relevé la relation foncière entre jeu et illusion - inlusio ou « entrée dans le jeu »-, la seconde étant à la fois condition et conséquence du premier. L'essence du jeu se situe ainsi dans cet équilibre entre illusion et lucidité - illusion d'être entièrement dans un autre monde et lucidité d'être cependant dans un espace fictif, d'où l'on perçoit mieux la gravité ou la monotonie de la vie réelle. Car c'est bien avec l'intention d'échapper momentanément à tout ce que la vie

1 Le terme jeu de mots, selon sa définition par Le Grand Robert de la langue française (2001) « allusion plaisante fondée sur l'équivoque de mots qui ont une ressemblance phonétique mais contrastent par le sens »-, est limité au seul jeu sur l'équivoque de sens engendrée par l'homonymie ou par la polysémie. Ainsi, le calembour, l'à-peu-près, la syllepse et l'antanaclase doivent constituer les formes principales du jeu de mots au sens strict. 
comporte de grave ou de banal que l'on s'adonne au jeu qui « repose » et qui «amuse » (Caillois 1967 : 9). Face tant aux difficultés qu'à la routine de l'existence, celui-ci constitue "une sorte de havre où l'on est maître du destin " (Caillois 1950 : 213), " un îlot de clarté et de perfection » (Caillois 1967 : 20) voué à l'abandon, l'aisance, la joie et la créativité. C'est dans cela que réside sa fonction.

Par conséquent, un langage placé sous le signe du jeu échappe à l'usage courant; il se cantonne dans une sphère illusoire et autonome, un mécanisme " artificiel » où il obéit, comme le souligne Todorov, à une règle particulière. ${ }^{2}$ Jouer avec les mots revient à s'écarter de la convention générale du langage - y compris de la logique qui préside aux règles linguistiques ; c'est en même temps prendre ses distances par rapport aux simples finalités de la signification et de la communication. Freud attribue le plaisir du travail du « mot d'esprit » au relâchement de la pression du réel, au défoulement émotionnel grâce à l'élimination d'inhibitions (Freud [1940] 1988 : 249). Le procédé du jeu de mots déborde des limites régulières de la langue, ce qui lui permet de prendre en compte la matière même des mots. Autrement dit, c'est " une dysfonction du langage » (Guiraud 1976 : 111) ou, comme le définissait déjà Bergson, une « distraction momentanée du langage » (Bergson [1900] 2011 : 125). Du fait de son caractère hors normes, un jeu de mots est forcément créatif et ludique ; il représente une sorte de havre où la dérive, la fantaisie et l'extravagance sont permises dans un esprit de défoulement et d'amusement.

La plaisanterie et le divertissement, dotés respectivement des fonctions amusante et reposante du jeu définies par Caillois, constituent pour nous les deux fondements du jeu de mots. ${ }^{3}$ Le premier, dominé par la paidia, a pour

2 «Le (jeu» des mots s'oppose à l'utilisation des mots, telle qu'elle est pratiquée dans toutes les circonstances de la vie quotidienne. Cette opposition ne concerne pas seulement le jeu et le sérieux mais aussi la parole dont la construction obéit à une règle particulière (parole artificielle) d'une part et, de l'autre, la parole qui ne sert qu'à exprimer, à désigner, à inciter, qui se consume dans sa finalité ou dans son origine (parole naturelle)» (Todorov 1978 : 294).

3 Guiraud, lui, divise les jeux de langage en deux genres : ceux de la plaisanterie qui « jouent sur des mots » (le calembour, la contrepèterie, l'anagramme, etc.) et ceux du divertissement qui " jouent avec des mots » (la charade, le rébus, les bouts-rimés, les mots croisés, etc.) (Guiraud 1976 : 97-98). Cependant, cette division est faite selon les types de jeux vus comme immuables ; or, c'est plutôt le contexte d'emploi qui détermine le caractère d'un jeu : la pratique de calembours ou de contrepèteries pourrait aussi bien être un divertissement entre amateurs de mots qu'une plaisanterie. Par ailleurs, les divertissements verbaux au sens strict, appelés aussi jeux d'esprit ou « jeux avec des mots » selon l'auteur, ne revêtent pas la nature du jeu de mots à notre sens, avec des relations différentes du joueur avec les mots dont il se sert comme un simple outil du passe-temps intellectuel sans être intéressé par leur propre mécanisme. Nous 
principe l'anarchie et montre des affinités avec la culture populaire : c'est de la désobéissance à la norme, de la transgression des règles, de l'opposition à l'ordre, quand ce n'est pas à la perfection, que provient le plaisir partagé. Le second, tendant vers le ludus, est d'ordre esthétique, voire spirituel : il est nourri par la volonté de construire de nouvelles règles, souvent difficiles, destinées à surpasser la facilité du langage ordinaire. Le jeu de mots ressortissant à la plaisanterie se moque de l'arbitraire des signes et de la sclérose des conventions verbales en les détournant, les ridiculisant ou les dégradant. Il constitue un infra-discours destructeur de la logique et du système grammatical ; ainsi revêt-il une fonction critique. Quant au jeu de mots relevant du divertissement, il déplace l'attention de l'aspect pragmatique du langage vers ses potentialités ludiques ou sa « fonction poétique » au sens jakobsonien. ${ }^{4}$ Explorant la richesse et la musicalité du signifiant, il est doté d'une fonction poïétique et constitue un super-discours qui fait de la langue un objet d'art.

\subsection{Le jeu de mots dans le théâtre traditionnel}

La construction-acceptation de l'illusion - le noyau du jeu - est essentielle dans tout système dramaturgique. De plus, selon le principe aristotélicien qui a régné pendant de longs siècles en Occident, l'illusion théâtrale - que Corneille appela très justement «l'illusion comique » - est fondée sur la mimésis. À l'époque classique, alors que celle-ci restait la règle dans les beaux-arts, elle fut contaminée au théâtre par le concept de vraisemblance, qui liait la crédibilité de la représentation à un rapport intellectualisé avec la réalité. Le jeu de mots, dans ce théâtre où la quête d'une illusion parfaite amenait l'auteur à composer un « bel animal ( (Aristote 1874: 1450b) cohérent, implique donc une double illusion : celle du verbe et celle de la vie. En d'autres termes, deux écarts se trouvent étroitement imbriqués: celui d'avec le langage usuel va de pair avec celui

\footnotetext{
proposons donc une division - toutefois non tranchée car nombreux sont les cas de fusion des jeux de mots selon leurs fondements : ceux fondés sur la plaisanterie et ceux fondés sur le divertissement. La plaisanterie n'est pas le synonyme du jeu de mots au sens strict, mais désigne tout jeu de langage qui plaisante avec la norme linguistique en la transgressant ; tout comme le divertissement ne renvoie pas ici aux jeux d'esprit, mais bien à l'idée d'explorer, en s'amusant avec, l'aspect ludique du langage.

4 «La visée (Einstellung) du message en tant que tel, l'accent mis sur le message pour son propre compte, est ce qui caractérise la fonction poétique du langage. [...] Cette fonction, qui met en évidence le côté palpable des signes, approfondit par là même la dichotomie fondamentale des signes et des objets... » (Jakobson [1963] $2003: 218$ ).
} 
d'avec la réalité, et tous deux fonctionnent de concert. Si ce second écart se résume, pour se borner au niveau verbal du langage dramatique, dans l'écart du discours dramatique vis-à-vis du discours ordinaire, le jeu de mots au thêâtre est alors un surcroît de jeu, un renforcement de cet écart d'avec le langage courant. Sont renforcés, précisément, les effets tragiques ou comiques contribuant à l'illusion dramatique et créés en grande partie par un langage distinct de l'usage.

Il faut cependant relever une différence entre les genres. Si le langage poétique de la tragédie classique est le résultat des tentatives de jeux de mots visant, à l'origine, à relever la musicalité de la parole, il a fini par devenir un code stylistique et a perdu sa nature ludique, à cause non seulement du besoin de fournir des règles au genre noble, mais aussi d'une recherche rigoureuse de la vraisemblance qui élimine toute possibilité de repousser les limites du langage. Au contraire, le genre comique, dont le langage imite pour tourner en dérision le parler mécanique, ridicule ou provincial, donne plus de liberté aux jeux de mots. Leur présence y est appréciée pour son apport à l'illusion dramatique, lorsqu'elle va dans le même sens caricatural du discours comique en en poussant encore plus loin le burlesque.

Il reste que l'illusion verbale ne doit jamais dépasser ni contrarier l'illusion dramatique : son rôle est de la servir et de la fortifier. Certes la surprise et le rire du spectateur réagissant à un jeu de mots signifient une sortie de l'illusion, mais l'adéquation - quasi immédiatement perçue - de ce jeu au fonctionnement du drame assure la réintégration du spectateur à l'histoire et la victoire du mimétisme. En revanche, la séparation de ces deux illusions, ou l'altération de leurs rapports d'entente ou de subordination, aurait menacé le maintien de l'illusion et le fonctionnement du thêâtre aristotélicien ; et c'est bien dans cette libération du jeu de mots de son assujettissement au drame que consiste un des enjeux du thêâtre moderne.

Le jeu de mots dans ce théâtre traditionnel est également caractérisé par la connivence entre auteur et public : cette finalité du jeu de mots qu'est la création d'une "connivence ludique» (Kerbrat-Orecchioni 2011) est d'autant plus marquée au théâtre, avec la présence directe des spectateurs en face des comédiens. De la plaisanterie burlesque sur la rigidité du langage, au divertissement esthétique pour un langage potentiel, quel que soit le niveau où il se situe, le jeu de mots n'est créé que pour être compris et apprécié d'un public, défini par une époque et un milieu. Tandis que le jargon inintelligible des farces médiévales est supposé s'adresser au goût populaire, au désir pur d'évasion que les spectateurs éprouvaient en allant au théâtre (Garapon 1957 : 98-101), l'à-peu-près de Molière devait cibler les "honnêtes hommes" de la Cour et de la Ville qui, 
réjouis de retrouver à la scène les valeurs de leur monde, étaient tout disposés à se gausser de qui les ignorait ou s'efforçait de les adopter - souvent en empruntant, de maladresse en maladresse, leur langage (Forestier et Bourqui 2010). Si, au siècle suivant, les mots d'esprit de Marivaux étaient accueillis d'abord par un public de salon qui, amateur de liberté et de raffinement verbaux, annonçait les Lumières (Deloffre 1989), les jeux de mots - au sens strict - de Feydeau allaient trouver leur bonne place dans le vaudeville, genre populaire dont le dessein primordial était de provoquer le rire en renvoyant au public sa propre bêtise et ses tics verbaux (Gidel 1979).

En somme, aucun jeu de mots n'était gratuit dans le théâtre traditionnel : tous étaient intégrés à la mécanique dramatique, pour souligner le comique de caractère (Molière), le comique d'idée (Marivaux) ou le comique de situation (Feydeau). Certes au Moyen Âge, où l'idée de drame était loin d'être aboutie, la fantaisie verbale dans les genres comiques s'arrêtait au comique des mots mêmes, mais elle était avant tout un stimulant de l'euphorie collective propre à l'ambiance de fête. Tous servaient au dramaturge de moyens pour renforcer le lien entre spectacle et spectateurs, indispensable au succès de la pièce, mais aussi pour réunir et souder les spectateurs.

\subsection{Le jeu de mots dans le théâtre d'avant-garde des années 1950}

Si la modernité en littérature et dans les arts consiste en un affrontement entre le réel et le langage, un refus du confort formel, des belles-lettres, de l'homogénéité sémantique et de l'utilité verbale, une volonté de refonder le langage et les représentations du monde, et encore le goût du renversement et de l'expérimentation, le thêâtre français signa sa venue à la modernité en 1896 avec la réalisation d'Ubu Roi. Depuis, «la volonté d'assumer et d'explorer les ressources de la thêâtralité, le refus du carcan de la représentation illusionniste » (Roubine 1980 : 16) ont délivré le théâtre de son obligation de vraisemblance, et préparé son autonomie par rapport au drame. Cette transformation a pris du temps : qu'il s'agisse de l'usage auto-réflexif des signes revendiqué par les dadaïstes et les surréalistes, ou du retour aux sources spectaculaires du théâtre réclamé par Artaud, dont la tentative avec Vitrac en 1928 a connu une froide réception ${ }^{5}$, les

5 Roger Vitrac, sans doute le seul de son temps à concrétiser le principe surréaliste dans la dramaturgie, est un précurseur du théâtre «non-figuratif » revendiqué par la génération des années 1950. Son Victor ou les Enfants du pouvoir, conçu pour le Théâtre Alfred Jarry qu'il a 
premières mises en pratique datent des années 1950. Elles ont eu pour cadres les petits théâtres parisiens de la rive gauche où s'élaborait alors une nouvelle dramaturgie anti-aristotélicienne, « ayant fini d'être dramatique » et quittant les "principes de narration et de figuration" (Lehmann 2002: 20). Le dialogue, avec la « crise du drame » (Szondi [1965] 2006) et la désactivation de la fonction communicative du langage dramatique, laissa la place aux échanges incohérents ou au monologue morcelé, à la logorrhée insignifiante ou au silence obscur.

Le jeu de mots dans ce théâtre épris d'autonomie s'avère à la fois un héritage et une subversion de son passé. D'une part, tout en développant l'illusion verbale dans les deux voies de la plaisanterie et du divertissement, il cesse d'être attaché à l'illusion dramatique; il accède ainsi à une liberté et une gratuité qui le relient, par-delà les siècles et tout ce qu'ils entraînent d'anachronique, à la tradition orale médiévale selon laquelle la théâtralité verbale (son, rythme, prosodie) peut constituer à elle seule l'action et le spectacle mêmes. D'autre part, les auteurs d'avant-garde, habités par une vision sceptique du langage et de la fonction théâtrale, ne cherchent plus à plaire aux spectateurs bourgeois pour la plupart - ni à les instruire ou les réunir, mais à les provoquer, les diviser, les faire réfléchir, en déjouant sans cesse leurs attentes en leur imposant le nonsense. ${ }^{6}$ Le rire de connivence suscité jadis par les jeux de mots se transforme souvent en un rire ambigu et précaire, déclenché par l'étrangeté des associations de mots et aussitôt figé par l'angoisse de ne pas en comprendre le sens. Dès lors, quoique les techniques restent les mêmes, le jeu de mots ne renforce pas l'illusion mimétique, ni la connivence dramatique avec le public ; il est au contraire la source première d'un nouvel écart - celui que le langage désormais théâtralisé prend vis-à-vis des conventions du langage dramatique.

fondé avec Artaud et monté par ce dernier en 1928 à la Comédie des Champs-Élysées, annonce aussi bien les dysfonctionnements du langage chez Ionesco que les paroles ludiques et oniriques chez Tardieu.

6 Pour éviter l'interprétation péjorative du mot non-sens, qui renvoie à un manque ou une absurdité de sens reprochable pour un esprit cartésien, nous empruntons le terme anglais nonsense qui, ne comportant pas de connotation négative, renvoie à une forme d'humour liée à l'absurdité ou à l'excentricité et relevant d'une tradition littéraire reconnue dans la culture anglo-saxonne. Trouvant ses formes primitives dans la littérature orale médiévale, dont la règle de jeu réside dans l'incohérence systématique de sens et dans la primauté du rythme sur la logique (cf. Mougin 2004 : 14), le nonsense est une synthèse de l'enfantillage et de l'oralité. C'est notamment après la Seconde Guerre mondiale que la France cartésienne a rouvert la porte à ce comique d'incongruité, accueilli d'abord dans les cabarets et les cafés-théâtres, où Ionesco et Tardieu puisaient leurs premières inspirations. 
Ce «théâtre nouveau » des années 1950 adopte deux types d'attitudes à l'égard du langage : d'un côté la dénonciation, le doute radical, « dont les implications ont une portée sociale et politique ou métaphysique »; de l'autre un esprit de confiance, de réconciliation, ayant recours à un langage poétique qui « cherche à briser les moules habituels de la phrase et de la rhétorique, moins pour étonner le spectateur que pour dire plus et autrement » (Corvin [1963] 1995 : 19-20). La même idée a amené Martin Esslin à opposer le «théâtre de l'absurde », celui de Beckett, de Ionesco et d'Adamov, à ce qu'il appelle le « théâtre de «l'avant-garde poétique» » (Esslin 1963 : 22), représenté notamment par Pichette, Schehadé, Tardieu, Audiberti. Cette division générale correspond à deux attitudes de l'avant-garde : l'une, négative, s'exprime par « un mélange [...] de gratuité et d'exaspération, de nihilisme et de protestation effrénée » (Marino 1986 : 659) tandis que l'autre, positive, dérive d'un « esprit profondément précurseur, ‘futuriste` par définition, largement ouvert sur l'avenir, fasciné par le grand «lendemain` » (Marino 1986: 721). La première, appliquée au langage, mène à la dégradation de la norme et à la rupture de la logique discursive ; elle relève de la plaisanterie en tant que principe anarchique. La seconde se voue à l'expérimentation du potentiel signifiant du mot et à l'enrichissement des moyens d'expression; sur le plan esthétique, elle est l'un des modes fructueux du divertissement.

\section{Deux exemples antithétiques : lonesco et Tardieu}

\subsection{La « tragédie du langage » et la « comédie du langage »}

Parmi les auteurs révolutionnaires cherchant à détourner les conformismes langagier et esthétique d'un théâtre en retard sur d'autres arts dans l'évolution vers l'autonomie, Eugène Ionesco et Jean Tardieu sont des figures de proue. Ils sonnent le glas de ce qui était perçu comme réaliste et annoncent la naissance d'un théâtre abstrait, éloigné tant du psychologisme que du rationalisme, remplacés par le dépassement et la reconstruction du réel. Tous deux sont des héritiers spirituels de l'esprit pataphysique de Jarry consistant à renverser le « consentement universel » par l'union des contraires (Jarry 1972 : 668-673), et font partie de la génération de Queneau, Vian et autres humoristes contestataires. Ce qui les distingue pourtant de cette génération, c'est qu'ils ont réalisé comme prenant la relève de Vitrac - les théories et les projets des avant-gardes, aussi bien dans l'écriture que sur la scène, si bien que la révolution du langage 
a dépassé la phase des essais ésotériques pour affronter le public et ébranler les conformismes dramatiques. Pour ce faire, ils visent tous les deux, au début de leurs carrières, le vaudeville comme cible de dérision, tout en puisant dans les thèmes, les situations voire les formes de jeux de mots fréquentés par un Feydeau ou un Labiche.

Cette obsession commune de la question du langage est omniprésente dans les œuvres des deux écrivains, qui témoignent, malgré les approches très différentes, d'une grande proximité dans les thèmes, les schémas, voire les dénouements. La Cantatrice chauve partage avec Un mot pour un autre le même cadre bourgeois, ses clichés de conversation et ses règles de jeu ; La Leçon et Ce que parler veut dire donnent la parole au même type de professeur arbitraire, qui manipule la langue et les dénominations; Jacques ou la Soumission et Les Temps du verbe réduisent l'homme à la proie du Verbe ; Les Chaises et Une voix sans personne laissent résonner des paroles décousues et répétitives sans destinataires; Les Salutations et Les Mots inutiles sont constitués à partir d'une logorrhée truffée de mots peu usités, alors que Le Maître et La Serrure montrent, à travers l'adoration d'une figure macabre muette, que la parole ne vaut pas le silence ; Le Salon de l'automobile et Le Style enfantin jouent sur le coq-à-l'âne propre au langage enfantin ; La Jeune Fille à marier et Monsieur Moi mettent un phraseur arrogant, qui est l'incarnation des clichés, en face d'une figure faible jouant son ombre ou son écho ; Scène à quatre ressemble à La Sonate et les trois messieurs en ce que les deux pièces font entendre un trio de voix masculines tournant autour du néant... Ce n'est donc pas un hasard si l'un et l'autre ont attiré les mêmes metteurs en scène d'avant-garde (Jacques Polieri, Sylvain Dhomme et Jorge Lavelli), les mêmes spectateurs enthousiastes (Queneau, Soupault, Paulhan, Lemarchand, Touchard), les mêmes critiques (Marcabru, Lerminier, Kemp), ou encore les mêmes chercheurs, dont Paul Vernois, qui a consacré à chacun une monographie (Vernois 1972, 1981). Cependant, tout nourris qu'ont été Ionesco et Tardieu à des sources communes, et si lancés qu'ils soient dans une commune recherche d'innovation théâtrale, leur chemins divergent, et cela dès le départ. Ils représentent les deux attitudes d'avant-garde précitées, à l'égard du langage en général et de l'esthétique dramatique : celle de la rupture $^{7}$ et celle de la réconciliation ${ }^{8}$. Ramenées aux jeux de mots, Ionesco y

7 Toute la vie de Ionesco est marquée par « un goût pour la polémique et une haine des idéologues de tout poil » (Jacquart 1998 : 20). Cette haine de toute incarnation de l'autorité est à l'origine de son combat constant contre les conformismes, combat qui procède par la plaisanterie sarcastique sur les clichés qu'emploient les autorités. De la satire des clichés et du langage automatique de la petite bourgeoisie dans La Cantatrice chauve (1950), Ionesco s'ache- 
cultive l'esprit de plaisanterie, tourné vers la destruction; Tardieu l'esprit de divertissement, déployé à des fins de construction.

Mû par la haine du théâtre de son temps, Ionesco s'inspire, dans ses « farces tragiques ${ }^{9}$, de la verve anarchiste de Jarry ou de l'Apollinaire des Mamelles de Tirésias. Faisant du « grossissement des effets » (Ionesco 1966 : 59) - procédé du guignol - le principe fondateur de son esthétique théâtrale, il pousse à l'extrême le mécanique et l'insolite pour donner au spectateur le «sentiment de l'étrangeté du monde " (Ionesco 1966 : 53). Sans aller jusqu'à pratiquer l'écriture automatique (dont la touche onirique est pourtant très présente dans ses œuvres), il fonde, dans la lignée des surréalistes, ses jeux de mots sur le hasard. C'est ainsi que dans La Cantatrice chauve, désignée par lui comme une «tragédie du langage », il déjoue la logique et la cohérence. Par l'emploi d'un nonsense dérisoire, qui expose ses personnages à l'échec du dialogue et noie ses lecteurs-spectateurs dans l'incompréhension, il s'attaque à l'automatisme de la pensée, exprimé par l'usage mécanique des clichés verbaux, et aux banalités creuses de la communication. Le comique verbal n'est chez lui qu'une étape

mine vers la dénonciation de l'épidémie idéologique dans Rhinocéros (1960). À force de s'attaquer aux dogmes autoritaires et aux courants majeurs de son temps - tels le brechtisme à travers ses créations de plus en plus "sérieuses » et « engagées », mais aussi ses polémiques avec des critiques, celui qui a défini l'avant-garde "en termes d'opposition et de rupture » (Ionesco 1966: 77), comme une minorité impopulaire, fondera en moins d'une décennie sa propre école, vénérée et suivie par beaucoup. Néanmoins, ce changement de position n’a pas empêché l'esprit dérisoire de dominer encore ses dernières pièces : le Bérenger de Rhinocéros est une incarnation même de la lucidité à l'égard des autorités et du doute tenace de la vogue. Qu'il soit de "l'anti-théâtre » ou du thêâtre, c'est "un théâtre de la dérision » (Ionesco 1966: 190) qu'il prétend faire, même si la dérision apparaît moins dans le langage même que dans le grotesque des « éléments dramatiques » (intrigue, situation, personnage) au fil du temps et de la consécration académique de l'auteur.

8 «L'expérimentation a été la constante de sa création dramatique » (Mignon 2003: 8). Guidé par cet esprit d'expérimentation d'un modeste « apprenti dramaturge » (Tardieu 1966: 8), Tardieu essaie de «fasciner (plutôt que de «convaincre») à partir des matériaux élémentaires dont [un poète] est bien obligé de se servir, c'est-à-dire les mots du langage », en « les [détournant] de leur fonction habituelle et [en] les [combinant], comme des couleurs ou des sons » (Tardieu 1978 : 134). Au lieu de prendre la position du rival contre le théâtre conventionnel de son temps, il rivalise plutôt avec la peinture et la musique par une écriture poétique tendant vers l'abstraction, un "para-théâtre » (Tardieu 2003 : 150), afin d'enrichir les fonds de la dramaturgie. Ses jeux de mots, s'ils rappellent encore les formes comiques « traditionnelles » dans ses premières pièces, consisteront entièrement en jeux de sonorités propres à la poésie, lorsque son «théâtre de chambre » (titre de son premier recueil de théâtre) évoluera vers une «musique de chambre » avec les mots transformés en pures notes musicales.

9 «Farce tragique » est utilisée par l'auteur comme le sous-titre des Chaises (1951). 
pour accéder à un tragique sous-jacent (Ionesco 1966 : 173), c'est ainsi que ses jeux de mots, ludiques certes, prennent une dimension critique.

$\mathrm{Au}$ contraire, c'est bien l'amour pour le théâtre en général qui amène Tardieu à la création dramatique, et c'est avec les Oulipiens, qui cherchent la liberté à partir des contraintes, qu'il se sent en affinité. L'homme n'est pas un destructeur des règles mais un constructeur, qui se dévoue à l'enrichissement du langage en établissant un " ‘catalogue` des possibilités théâtrales » (Tardieu [1966] 1987 : II) ouvert aux expérimentations, comme Queneau quand il propose ses «exercices de style». D’où le petit format de la plupart de ses pièces, empreintes de surcroît de la forme radiophonique qui travaille avant tout sur la matière sonore des mots. ${ }^{10}$ Nommant un de ses recueils « comédie du langage » (Tardieu [1966] 1987 : II), où domine un nonsense poétique créé par les à-peu-près, les mots "sauvages » (Tardieu 1966 : 168), les "somniloquies» décousues et le silence, il invite les lecteurs-spectateurs à un colin-maillard du langage en les informant des "règles du jeu » au lever du rideau. Derrière le comique et le plaisir liés au son, c'est la potentialité du signifiant qu'il explore, en empruntant des moyens d'expression à la musique, jusqu'à créer des « infralangages » des sons (Tardieu 1978 : 13) qui fonctionnent en deçà de l'expression discursive. Ses jeux de mots prennent ainsi une dimension poïétique.

Chez Ionesco, les mots sont des carcans à casser ; chez Tardieu, ce sont des instruments cachés. Ces deux conceptions du jeu verbal, tant sur le plan paradigmatique (la substitution) que sur le plan discursif (l'enchaînement) - pour emprunter la catégorisation proposée par Guiraud (Guiraud 1976) -, appellent une comparaison.

\subsection{La substitution : un chaos sémantique face à un jeu d'énigmes}

«L'équivoque constitue l'essence du jeu de mots » (Guiraud 1976 : 9). Sa forme classique se fonde sur le remplacement d'un (des) terme(s) par un (des) terme(s) qui lui est ou sont phonétiquement analogique(s), de manière totale ou approxi-

10 La vie et l'œuvre de Tardieu sont profondément marquées par ses longues années d'expériences radiophoniques : sa prise en charge en 1946 des programmes culturels, notamment du Club d'Essai à l'Office de la Radio-Télévision Française, marque sa venue officielle au thêâtre, genre qui, pour lui, remédie à l'inefficacité de la poésie grâce à une parole totale. Dès lors, il se sert des ondes comme d'une scène pour approfondir son exploration des aspects sonores de la langue française et poursuivre sa recherche de formes théâtrales. 
mative. Dans ce cadre, la syllepse et l'antanaclase (reposant sur la polysémie), comme le calembour et l'à-peu-près (reposant sur l'homophonie) sont les formes principales de substitution. On peut légitimement y ajouter la néologie, qui consiste la plupart du temps à substituer à un terme jugé usé (et, par là, faiblement expressif), un autre, inédit, né a priori d'une combinaison (mot-valise) ou d'un exercice de l'ordre de la dérivation.

\subsubsection{Syllepse vs. à-peu-près}

Tandis que la syllepse et le calembour classiques s'appuient sur le glissement sémantique pour provoquer la surprise et le rire, l'à-peu-près, qui voile une partie du signifiant attendu sans pour autant détourner le sens global du propos, surprend et amuse plutôt par l'énonciation que par l'énoncé. Dans le thêâtre traditionnel, les deux premières figures sont souvent utilisées comme détonateurs d'un conflit, tournants d'une situation, ou pis-aller de la défense dans un affrontement verbal ; elles servent de stimulants au dramatique et de ressorts au comique. Quant à l'à-peu-près, qui est moins un enjeu du dialogue qu'un assaisonnement, il participe surtout du pastiche ou de la caricature; visant à souligner la maladresse de celui qui parle par rapport à toute espèce de norme (usage courant ou marque de distinction), il inclut le balbutiement, le lapsus, la confusion, aussi bien que les accents ruraux ou exotiques...

Or, ces trois formes de jeu ne revêtent pas les mêmes fonctions chez nos deux auteurs. Si Ionesco se sert fréquemment de la syllepse et du calembour, c'est pour paralyser la logique et casser la cohérence en entraînant le glissement sémantique jusqu'à l'impasse du non-sens; et si Tardieu a une prédilection pour l'à-peu-près, au point de le pousser jusqu'à sa forme extrême (un-mot-pourun-autre), c'est que ce procédé implique à ses yeux un déchiffrement heuristique du "palimpseste» (Genette 1982: 57-62) qui lui permet de tourner en ridicule les habitudes verbales des bourgeois. Lisons le premier, dans La Cantatrice chauve et dans Jacques ou la Soumission :

(1) Madame Smith : Dommage ! Il était si bien conservé.

Monsieur Smith : C'était le plus joli cadavre de Grande-Bretagne ! Il ne paraissait pas son âge. Pauvre Bobby, il y avait quatre ans qu'il était mort et il était encore chaud. Un véritable cadavre vivant... (Ionesco 1991 : 12)

(2) Prenez un cercle, caressez-le, il deviendra vicieux ! (Ionesco 1991 : 39) 
(3) Jacques : Elle n'est pas moche! Elle n'est pas moche! Elle ne fait même pas tourner le lait ${ }^{11}$... elle est même belle...

Roberte mère : As-tu du lait ici pour voir?

Roberte père : Il ne veut pas, il bleufe. Il sait que le lait tournerait... (Ionesco 1991 : 103)

(4) Jacques père : Il est sourd et muet. Il est chancelant.

Jacqueline : Il chante, seulement. (Ionesco 1991 : 89)

(5) Roberte II : ...On m’appelait aussi l'aînée gaie...

Jacques : À cause de vos nez?

Roberte II : Mais non. C'est parce que je suis plus grande que ma sœur... ${ }^{12}$

(Ionesco $1991:$ 106)

Contrairement à ces dérapages sémantiques, cocasses et absurdes, ceux de Tardieu sont phonétiques, ironiques et ludiques : l'auteur badine avec les conversations creuses en remplaçant à son gré un mot par un autre. Son jeu respecte quelquefois le principe de l'à-peu-près, par approximation phonétique : «En été, poursuivre le plumier [pluvier] des marais, rester des heures dans la boue, à l'affût d'un buvard [busard/bouvard] sauvage, en automne, forcer la cheviotte [chevrette] à la course » (Tardieu 1966 : 181) ; "chatoyer [chatouiller] les naseaux de la Baronne » (Tardieu 1966 : 184) ; "il me serre dans ses draps [bras], contre son moteur [cœur]» (Tardieu 1975: 89). Ailleurs, l'approximation est sémantique : «il ne fait rien de ses dix bras [doigts] » (Tardieu 1975 : 89) ; "Mon chéri ne fera pas longtemps le pied de mouton [grue] sur le quai de la station » (Tardieu 1966: 184). D’autres fois, le jeu est plus aléatoire; il ne suit ni le principe strict de l'à-peu-près, ni celui du pastiche phraséologique relatif au contexte, ni la règle d'une espèce de jeu de société où une énigme n'admettrait qu'une seule réponse. La substitution introduit des mots peu fréquents, parfois sans relation avec ceux qu'ils remplacent. Les exemples ne manquent pas dans une de ses pièces les plus représentées et aussi les plus étudiées, Un mot pour un autre: «Mes trois plus jeunes tourteaux ont eu la citronnade, l'un après l'autre. Pendant tout le début du corsaire, je n'ai fait que nicher des moulins, courir chez le ludion ou chez le tabouret, j'ai passé des puits à surveiller leur carbure... $»^{13}$ (Tardieu [1966] 1987: 14); "Mon zébu semble tellement à ses

11 La phonétique de laid - synonyme de moche - rappelle son homophone lait.

12 Jacques, résistant au mariage imposé par ses parents, réclame une femme à trois nez, et voilà qu'il tombe sur Roberte II, qui a exactement trois nez. Elle est la grande sœur de Roberte, la fiancée proposée que Jacques trouve laide car elle n'a que deux nez. Ici, à la suite du calembour entre l'aînée et les nez, l'auteur détourne aussi le sens de grande.

13 Alain Deligne a « traduit » la citation par « Mes trois plus jeunes enfants ont eu la jaunisse, l'un après l'autre. Pendant tout le début de l'hiver, je n'ai fait que chercher des médicaments, 
planches dans votre charmant tortillon... que l'on croirait... oserais-je le moudre ? ${ }^{14}$ (Tardieu [1966] 1987 : 17). Quant aux sobriquets entre les amants, ils sont ouverts à toutes les traductions possibles, d'où la valeur pédagogique de ses pièces : " Je suis sa mouche, sa mitaine, sa sarcelle; il est mon rotin, mon sifflet ; sans lui je n'en peux ni coincer ni glapir » (Tardieu [1966] $1987: 15){ }^{15}$

\subsubsection{Néologismes gratuits vs. « mots sauvages »}

Non contents d'exploiter le contenu des dictionnaires, nos deux auteurs inventent des néologismes. Là aussi, chacun se montre créateur à sa manière.

Ionesco, dans Jacques ou la Soumission et L'avenir est dans les œufs, déforme les mots à la manière du parler d'Ubu, en employant mononstre pour monstre, Je t'exertre pour Je t'exècre, plauvre pour pauvre, orordre pour ordre (Ionesco 1991 : 88-131). Il fabrique des mots-valises complètement gratuits, tout à la fois libérés du principe de fusionner les sens de leurs composantes et sans rapport avec le contexte : octogénique, vilenain, égloge, aristocrave, praticide, abracante, flaute, cordoléances... (Ionesco 1991 : 88-125). Dans Les Salutations, il juxtapose des adverbes inédits et vides de sens, dérivés de termes scientifiques - dont la plupart sont des termes pathologiques, désagréables à l'oreille - pour prolonger des salutations fades : adénitemment, arthritiquement, bissextilement, cartilagineusement, cirrhosiquement, dégobillationneusement, dodécaédriquement, empuatissamment, gangréneusement... (Ionesco 1991 : 80-82).

Tardieu, quant à lui, invente des «mots sauvages » (Tardieu 1978 : 117) onomatopée, gazouillement enfantin, rugissement animal, idiolecte intime ou " patois des familles » - qu'il met à la place des mots usités et auxquels il attribue des significations fantaisistes. Son objectif est de construire la « langue Moi ${ }^{16}$ chère au professeur Frœppel, personnage campé à sa propre image :

\footnotetext{
courir chez le médecin ou chez le pharmacien, j'ai passé des nuits à surveiller leur température... » (Deligne 1991 : 96). Mais ce n'est évidemment pas la seule possibilité de traduction, comme d'ailleurs les citations précédentes (ainsi ludion n'a rien de commun avec médecin, ni tabouret avec pharmacien).

14 Nous pouvons « traduire » la citation par « Mon mari semble tellement à son aise dans votre charmant pavillon... que l'on croirait... oserais-je dire? ».

15 Alain Deligne a « traduit » la citation par « Je suis sa bouche, son refuge, son esclave; il est mon soutien, ma raison d'être ; sans lui je n'en peux ni vivre, ni exister » (Deligne 1991 : 98).

16 «Construisons la langue moi! La seule qui soit universelle! la seule qui ne serve pas à déguiser la pensée, la seule qui soit pure effusion, dialogue direct et immémorial entre le sujet et l'objet, entre la créature et Dieu !» (Tardieu $1978: 22$ ).
} 
(6) galops des dadas, furie des zizis, boum-boum des tam-tams, papattes des bêbêtes, piques des coupe-kikis, hurlements des totos et niam-niams, ondulement des chichis, des dondons, et clic et clac et bing et crac (Tardieu [1966] 1987 : 55)

(7) Le coco est au rococo ce que le kiki est au rikiki. (Tardieu [1966] 1987 : 57)

(8) Toutou a mazé fiture. ${ }^{17}$ (Tardieu [1966] 1987 : 59)

(9) Coba, pas cavaillé ! A fait bla-bla poum-poum avé les plouplous du tralala !18 (Tardieu [1966] $1987: 61$ )

(10) L'Amateur de journaux : Tioc, tioc, tioc, tioc, tioc, tioc, politique...

Lui : Beu, beu, beu, beu, beu, bonnes nouvelles?

L’Amateur : Dac, dac, dac, dac, dac, dac, mauvaises nouvelles ! [...] (Tardieu 1969 : 40)

Il fabrique aussi des mots étrangers dont les sons assument à eux seuls le dialogue : «Gouch! Gouch! Mogok! Bézoui bélek? Péché-pi, coto-cox?», «Evéhi, rémé-ha, ho hai-ho : oui dou you, lémé oui !... Mada-oua, a-da-doua, éré-oui » (Tardieu 1969 : 29).

\subsection{L'enchaînement : un défi à la raison face à une symphonie de sons}

Par rapport aux formes précitées de jeux de mots au niveau paradigmatique, fréquentées déjà dans le théâtre comique traditionnel, les jeux de mots au niveau discursif coïncident mieux avec l'irruption de la modernité, rappelant les souvenirs - certes théoriques - de "l'incohérence systématique » (Garapon 1957 : 48) propre à la fatrasie ou aux menus propos, composés sous la seule contrainte de la rime. Selon Guiraud, quand l'enchaînement des mots ne relève ni de la cohérence sémantique, ni des règles logiques, quand il devient gratuit et éventuellement cocasse ou absurde, il tombe dans le jeu de mots (Guiraud 1976 : 27). La volonté, déjà soulignée, de rompre avec les usages de la logique discursive et les lois de la communication, amène Ionesco à se laisser emporter par les enchaînements d'idées. De son côté, Tardieu puise dans les enchaînements de sons pour « faire bouger le langage » (Tardieu 1975 : 42) et expérimenter la musicalité des mots. Si ces deux types d'enchaînement ont en commun l'arbitraire et

17 «Le chien a mangé de la confiture » (« traduction » fournie dans le texte même).

18 «Comment! Je n'ai pas travaillé! Je n'ai pas cessé de parler et de discuter avec les plus importants délégués du Comité ! » (« traduction » fournie dans le texte même). 
de l'absurde, le premier, au fond, relève du désir de dérision et le second de la préoccupation poétique.

\subsubsection{Enchaînement par automatisme}

De temps en temps, Ionesco fait appel à ce que nous appellerons des «motstremplins » pour dérouter le dialogue vers l'image immédiate que la seule sonorité, détachée du sens qui vient d'être énoncé, évoque en lui par association des idées. Tout se passe alors comme s’il distrayait ses personnages des joutes verbales pour les embarquer dans un jeu de société indépendant de leur dialogue. Les personnages, réduits à des pantins passifs, semblent être manipulés par les mots : " au lieu que les hommes se servent du langage pour penser, c'est le langage qui pense pour eux » (Doubrovsky 1960 : 321).

(11) Le Pompier : « ... Le veau fut donc obligé de se marier avec une personne et la mairie prit alors toutes les mesures édictées par les circonstances à la mode. »

Monsieur Smith : À la mode de Caen.

Monsieur Martin : Comme les tripes. (Ionesco 1991 : 32)

(12) Le Pompier : « ... qui avait fait, à la fille d'un chef de gare, un enfant qui avait su faire son chemin dans la vie... "

Madame Smith : Son chemin de fer...

Monsieur Martin : Comme aux cartes. ${ }^{19}$ (Ionesco 1991 : 34)

(13) Jacqueline : Écoutez-moi, mon cher frère, cher confrère, et cher compatriote, je vais te parler entre deux yeux frais de frère et sœur. [...] mais que veux-tu, tant pis aller...

(Ionesco 1991 : 91)

(14) Jacqueline: Tu ne comprends pas que je suis envoyée vers toi, comme une lettre à la poste, timbrée, timbrée par mes voix aériennes, bon sang !

Jacques : Hélas, bon sang ne peut mentir ! (Ionesco 1991 : 91-92)

Chez Tardieu, le coq-à-l'âne que produit l'enchaînement par automatisme paraît plus poétique qu'absurde. Les dialogues des amoureux, chers à l'auteur, se déroulent souvent au fil de mots pris volontairement au pied de la lettre, comme si les personnages se livraient à un exercice de complicité dont l'enjeu consistait à réagir non au sens que dégagent les mots, mais bien à une parole ontologique cachée dans ces mots mêmes :

19 Le « chemin de fer » est un jeu de cartes. 
(15) Lui, commençant à s'échauffer : Et moi, je suis ce que je suis à la fin !

Elle, relevant le défi : Ah, tu es ce que tu es ! Eh bien, moi aussi !

Lui : Mais non, voyons ! Tu ne peux pas être ce que je suis !

Elle, pleine d'un reproche passionné, presque au bord des larmes: Je l'étais bien, tout à l'heure ! J'étais ton « je suis », tu étais mon « tu es ». Ah, tout est bien différent !

(Tardieu $1969: 27$ )

Quant au monologue, il lui permet, dans une forme assez lyrique pour s'approcher de la poésie, d'enchaîner en superposant les mots appelés et rappelés l'un par l'autre sans interruption, jusqu'à former un flux poétique :

(16) Depuis que tu es entrée dans ce tableau,

je te trouve belle

très belle

belle comme une pomme

comme une pomme de terre

comme une pomme de terre de feu

comme une pomme de terre de feu de bois.

(Tardieu 1969 : 279)

(17) [...] pichet du haut en bas fendu mon pichet de

terre

de terre cuite à point, de terre de pomme, de

terre de feu

de terre mienne, de terre brûlée de terre à bois de terre

à moi. ${ }^{20}$ (Tardieu 1969 : 280)

(18) Je n'ai jamais vu fabriquer les filins d'acier pour les navires... (Bâillant et s'étirant.)

... Mais j'ai vu se déployer des voiles par temps clair! (Un temps. Il se lève à moitié et s'assied sur le bat-flanc.)

... Je ne suis jamais entré dans les offices où l'on mesure le temps qu'il fera...

(Tardieu 1969 : 91)

\subsubsection{Enchaînement par homophonie}

Ionesco enchaîne des mots assonants ou allitératifs, appartenant à des domaines très différents, pour rythmer et faire évoluer les dialogues asémantiques en même temps que pour assurer le comique d'incongruité. Il pratique la paronomase aux seules fins de générer l'insolite : « on s'empêtre entre les pattes

20 Les deux citations sont tirées des Trois personnes entrées dans des tableaux. L'auteur y fait allusion à un tableau de Braque. 
du prêtre », «nous avons passé un vrai quart d'heure cartésien », "Quand on s'enrhume, il faut prendre des rubans », «Plutôt un filet dans un chalet, que du lait dans un palais », «Bazar, Balzac, Bazaine!», «En somme, vous n'auriez rien à craindre, c'est le crâne de la crème ! (Ionesco 1991 : 35-41, 98). Ailleurs, il multiplie, en un tempo accéléré, des paronymes de sens contradictoires: «C'est encore moi qui te privais de dessert, qui t'embrassais, te soignais, t'apprivoisais, t'apprenais à progresser, transgresser, grasseyer " (Ionesco 1991 : 88). Il lui arrive également de dérouler en litanie des termes spécifiques ou néologiques, partageant le même suffixe ou la même racine, mais sémantiquement éloignés : « Des marxistes. Des marquis, des marks, des contre-marks. » (Ionesco 1991 : 136), « Ça va... adénitemment, arthritiquement, astéroïdemment, astrolabiquement, atrabilairement, balalaïkemment, baobabamment, basculamment... » (Ionesco 1991 : 80).

L'enchaînement par homophonie est plus fréquent chez Tardieu, qui a tendance à faire de ses pièces des poèmes ou des concertos, dont l'essentiel réside dans l'harmonie et la réitération. Dans L'A.B.C. de notre vie, "symphonie sans musique, faite de paroles, de cris, de murmures» (Tardieu 1969: 71), Monsieur Mot et Madame Parole, comme deux partitions accompagnant celle du Protagoniste, s'enchaînent avec des mots allitératifs :

(19) Aboiement ! Abandon! Abolition! Bombardement ! (Tardieu 1969 : 78)

(20) Monsieur Mot : Bondir ! Battre !

Madame Parole : Balayer ! Briser !

Monsieur Mot : Barrage ! Bastion!

Madame Parole : Bouilloire ! Bifteck !

Monsieur Mot : Bâcher ! Bêcher ! (Tardieu 1969 : 102)

(21) Dévaluation !... Désacralisation! Détachement ! Disjonction! Discrimination! Dissémination! Ding, ding, don! Danse du ventre! Dormez sur vos deux oreilles! Délivrez-nous du mal !... (Tardieu 1969 : 113)

Ces paronomases, qui n'ont pas une plus grande cohérence que celles de Ionesco, produisent un autre effet : les mots étant transformés en notes musicales et intégrés dans le rythme et la mélodie du discours, l'auditeur-spectateur entend moins l'amalgame sémantique que l'accord harmonieux ou la percussion rythmique des signifiants. Au lieu de déjouer la logique pour faire fi de la raison, Tardieu raisonne avec la musique pour créer des associations inédites de sons. 


\subsubsection{Enchaînement aléatoire}

Si les deux premiers types d'enchaînement s'appuient encore sur quelque parenté entre les mots, cette dernière catégorie n'en inclut aucune ; elle se laisse dominer par le hasard. C'est ici que les quêtes de nos auteurs sont véritablement menées à leur terme. Ainsi, Ionesco fabrique des répliques dans le style du « cadavre exquis » des surréalistes, qui tournent la plate structure grammaticale en ridicule tout en la respectant à la rigueur, pour ne formuler finalement que des paralogismes loufoques ou des assertions irrationnelles :

(22) Je te donnerai les pantoufles de ma belle-mère si tu me donnes le cercueil de ton mari. (Ionesco 1991 : 39)

(23) L'automobile va très vite, mais la cuisinière prépare mieux les plats. (Ionesco 1991 : 40)

(24) Viens, Jacqueline, toi qui, seule, as suffisamment de bon sens pour ne pas te frapper dans les mains. (Ionesco 1991 : 91)

Tardieu, qui ne lance pas ce type de défi à la raison et à la logique, préfère inonder nos oreilles engourdies d'un «déluge de mots " ou d'un " océan de signes » (Tardieu 1975 : 34), ou encore d'une divagation onirique. Sa visée est de les réhabituer à la richesse ou au chaos des sons que l'homme a entendus quand il est venu au monde et dont il n'entend plus que le contenu passé l'enfance. Étant donné que tous les mots imaginables sont englobés dans cet immense univers de sons, le caractère aléatoire du discours trouve ainsi sa justification, de l'ordre de la poïétique :

(25) La voix d'enfant, implacable, martelant les mots avec indifférence et cruauté: La pitié, l'ordure, le précipice, la contrainte, l'éloquence, le ministre, la fête, la torture, l'opium, le bagne, la tendresse, le mouchard, le proton, le garçon d'étage, la luxure, le Discours de la Méthode, le Principe d'Incertitude, la pollution, le plastique, l'énergie atomique, le Vol du Bourdon, la Ronde de Nuit, l'huile de ricin, l'aménagement du territoire...

(Tardieu 1975 : 35)

(26) Choriste homme, avec une sorte de contentement : C'est une belle saison! Choriste homme, criant à tue-tête : J'étouffe !... Ouvrez donc les fenêtres !

Choriste femme, éclatant de rire: Je... Je ne vous reconnaissais pas !... Ce manteau de berger !... [...]

Choriste homme, à voix haute, comme annonçant une bonne nouvelle à des gens situés loin de lui : Les masques sont prêts !... Vous pouvez appeler les musiciens ! (Tardieu 1969 : 83) 


\section{Conclusion}

Le jeu de mots dans le théâtre des années 1950 ne fonctionne plus comme un renforcement des effets illusionnistes (comiques la plupart du temps) et cesse d'être un simple accessoire du comique d'idée, de situation ou de caractère ; retrouvant sa gratuité et son indépendance par rapport à la mimèsis et au drame, il contribue à l'autonomisation théâtrale. Empruntant des procédés anciens, les dramaturges renversent pourtant la «face » comique et expressive du jeu de mots - sa part utile à la communication -, pour montrer son « revers » insolite et obscur - sa part inutile voire néfaste au fonctionnement verbal. Ils en font ainsi le moyen, particulièrement efficace, de la subversion de l'ordre et l'outil d'une ample rénovation de l'esthétique théâtrale.

Voulant échapper aux conformismes bourgeois de l'après-guerre et proposer un théâtre non figuratif, Ionesco et Tardieu, représentant l'un l'attitude négative de la rupture et l'autre l'attitude positive de la réconciliation de l'avantgardiste avec le langage, ont recours à deux types de jeux de mots, qui, ayant certes des tangences entre eux, se fondent respectivement sur les principes de la plaisanterie et du divertissement. Au niveau paradigmatique, Ionesco mélange les signifiés et bouleverse la logique pour exposer son public à un chaos sémantique à travers les syllepses, les calembours et les néologismes gratuits, tandis que Tardieu remanie les signifiants et ébranle les images qu'évoquent les mots au moyen d'à-peu-près et des mots idiolectaux, sources d'un jeu constant d'énigmes. Au niveau discursif, les enchaînements d'idées chez Ionesco ridiculisent le mécanique et la vacuité de la parole communicative, alors que les enchainements de sons chez Tardieu sont voués à une sacralisation - par la musique - de la parole quotidienne et de la langue même qui pourrait signifier par les seuls sons. Ainsi, le jeu de mots a pris une dimension sarcastique et critique chez Ionesco et une dimension poétique et poḯtique chez Tardieu.

Il reste à évoquer les mises en scène que leurs pièces ont inspirées : ce sont elles qui ont permis à leurs jeux de mots de franchir l'enclos linguistique pour devenir des phénomènes scéniques. Pour le théâtre de dérision de Ionesco, du scandale provoqué par la première et canonique Cantatrice chauve au Théâtre de la Huchette aux multiples réalisations d'aujourd'hui, le jeu de mots, retravaillé à chaque fois selon la cible de la parodie choisie par le metteur en scène, variable de surcroît selon l'époque, ne cesse de remplir un rôle éminemment social. Quant aux jeux de mots de Tardieu, dont le répertoire oscille entre le comique et le poétique, ils lui ont valu aussi bien des succès au cabaret que des échecs dans les salles d'avant-garde, avec les expérimentations de «spectacle total » de Jacques Polieri. Aujourd'hui, entre les salles de cours, où Un mot pour 
un autre devient un matériel pédagogique pour l'enseignement du français, et les salles de théâtre contemporaines, où la forme poétique prolifère, le jeu de mots tardivien se voit lui aussi prolongé et renouvelé.

\section{Références bibliographiques}

Aristote. 1874. La Poétique. Edition d’Auguste Noël. Paris : J. Delalain.

Bergson, Henri. [1900] 2011. Le rire. Paris : Editions Payot \& Rivages.

Caillois, Roger. 1950. L'homme et le sacré. Paris : Gallimard.

Caillois, Roger. 1967. Les jeux et les hommes. Paris : Gallimard.

Corvin, Michel. [1963] 1995. Le théâtre nouveau en France. Paris : Presses Universitaires de France.

Deligne, Alain. 1991. Traduire le traduit. A propos d'iUn mot pour un autre) de Jean Tardieu. Bonn : Romanistischer Verlag.

Deloffre, Frédéric. 1989. Introduction. In Marivaux, Théâtre complet, Frédéric Deloffre \& Françoise Rubellin (éds.), I-XVI. Paris : Bordas.

Doubrovsky, Julien Serge. 1960. Le rire d'Eugène Ionesco. La Nouvelle Revue Française 86. 313-323.

Esslin, Martin. 1963. Le Théâtre de l'absurde. Traduit par Marguerite Buchet, Francine Del Pierre \& France Frank. Paris : Buchet-Chastel.

Forestier, Georges \& Claude Bourqui. 2010. Introduction. In Molière, Fuvres complètes, Georges Forestier \& Claude Bourqui (éds.), t. I, XI-LX. Paris : Gallimard.

Freud, Sigmund. [1940] 1988. Le mot d'esprit et sa relation à l'inconscient. Traduit par Denis Messier. Paris : Gallimard.

Garapon, Robert. 1957. La fantaisie verbale et le comique dans le théâtre français : du Moyen Age à la fin du XVIIe siècle. Paris : Armand Colin.

Genette, Gérard. 1982. Palimpsestes. Paris : Seuil.

Gidel, Henry. 1979. Le théâtre de Georges Feydeau. Paris : Klincksieck.

Guiraud, Pierre. 1976. Les jeux de mots. Paris : Presses Universitaires de France.

Huizinga, Johan. 1951. Homo ludens. Essai sur la fonction sociale du jeu. Traduit par Cécile

Seresia. Paris : Gallimard.

Ionesco, Eugène. 1966. Notes et contre-notes. Paris : Gallimard.

Ionesco, Eugène. 1991. Théâtre complet, Emmanuel Jacquart (éd.). Paris : Gallimard.

Jacquart, Emmanuel. 1998. Le théâtre de dérision : Beckett, Ionesco, Adamov. Paris : Gallimard. Jakobson, Romain. [1963] 2003. Essais de linguistique générale I. Les fondations du langage. Traduit par Nicolas Ruwet. Paris : Editions de Minuit.

Jarry, Alfred. 1972. Éléments de pataphysique. In Jarry, Euvres complètes, Michel Arrivé (éd.), t. I, 668-673. Paris : Gallimard.

Kerbrat-Orecchioni, Catherine. 2011. De la connivence ludique à la connivence critique : jeux de mots et ironie dans les titres de Libération. In Maria Dolores Vivero Garcia (éd.), Humour et crises sociales. Regards croisés France-Espagne, 117-150. Paris : L'Harmattan.

Lehmann, Hans-Thies. 2002. Le Théâtre postdramatique. Traduit par Philippe-Henri Ledru. Paris : L’Arche. 
Marino, Adrian. 1986. Chapitre IV Tendances esthétiques. In Jean Weisgerber (éd.), Les Avantgardes littéraires au XXe siècle, Vol. II, 633-791. Budapest : Akadémiai Kiadó.

Mignon, Paul-Louis. 2003. Un itinéraire théâtral. In Jean Tardieu, L'Amateur de théâtre, PaulLouis Mignon \& Delphine Hautois (éds.), 8-12. Paris : Gallimard.

Mougin, Sylvie. 2004. Du non-sens au nonsense : en guise d'introduction. In Sylvie Mougin \& Marie-Geneviève Grossel (éds.), Poésie et rhétorique du non-sens : littérature médiévale, littérature orale, 13-23. Reims : Presses Universitaires de Reims.

Robert, Paul (éd.). 2001. Le Grand Robert de la langue française : dictionnaire alphabétique et analogique de la langue française, Vol. 4. Paris : le Grand livre du mois.

Roubine, Jean-Jacques. 1980. Théâtre et mise en scène 1880-1980. Paris : Presses Universitaires de France.

Szondi, Peter. [1965] 2006. Théorie du drame moderne. Traduit par Sibylle Muller. Belval : Circé.

Tardieu, Jean. 1966. Théâtre de chambre. Paris : Gallimard.

Tardieu, Jean. 1969. Poèmes à jouer. Paris : Gallimard.

Tardieu, Jean. 1975. Une soirée en Provence ou Le mot et le cri. Paris : Gallimard.

Tardieu, Jean. 1978. Le Professeur Frœppel. Paris : Gallimard.

Tardieu, Jean. [1966] 1987. La comédie du langage. Paris : Gallimard.

Tardieu, Jean. 2003. L'Amateur de théâtre, Paul-Louis Mignon \& Delphine Hautois (éds.). Paris : Gallimard.

Todorov, Tzvetan. 1978. Les genres du discours. Paris : Editions du Seuil.

Vernois, Paul. 1972. La Dynamique théâtrale d'Eugène Ionesco. Paris : Klincksieck.

Vernois, Paul. 1981. La Dramaturgie poétique de Jean Tardieu. Paris : Klincksieck.

Winnicott, Donald W. 1975. Jeu et réalité. Traduit par Claude Monod \& Jean-Baptiste Pontalis. Paris : Gallimard. 